\title{
Runtime Energy Consumption Estimation for Server Workloads Based on Chaotic Time-Series Approximation
}

\author{
ADAM WADE LEWIS, NIAN-FENG TZENG, and SOUMIK GHOSH, \\ University of Louisiana at Lafayette
}

This article proposes a runtime model that relates server energy consumption to its overall thermal envelope, using hardware performance counters and experimental measurements. While previous studies have attempted system-wide modeling of server power consumption through subsystem models, our approach is different in that it links system energy input to subsystem energy consumption based on a small set of tightly correlated parameters. The proposed model takes into account processor power, bus activities, and system ambient temperature for real-time prediction on the power consumption of long running jobs. Using the HyperTransport and QuickPath Link structures as case studies and through electrical measurements on example server subsystems, we develop a chaotic time-series approximation for runtime power consumption, arriving at the Chaotic Attractor Predictor (CAP). With polynomial time complexity, CAP exhibits high prediction accuracy, having the prediction errors within 1.6\% (or 3.3\%) for servers based on the HyperTransport bus (or the QuickPath Links), as verified by a set of common processor benchmarks. Our CAP is a superior predictive mechanism over existing linear auto-regressive methods, which require expensive and complex corrective steps to address the nonlinear and chaotic aspects of the underlying physical system.

Categories and Subject Descriptors: B.8 [Hardware]: Performance and Reliability; C.4 [Computer Systems Organization]: Performance of Systems; D.4.8 [Operating Systems]: Performance

General Terms: Measurement, Performance, Reliability

Additional Key Words and Phrases: Analysis of variance, chaotic time series, energy consumption, HyperTransport buses, performance counters, QuickPath links, thermal envelope, time series approximation

\section{ACM Reference Format:}

Lewis, A.W., Tzeng, N.-F., and Ghosh, S. 2012. Runtime energy consumption estimation for server workloads based on chaotic time-series approximation. ACM Trans. Architec. Code Optim. 9, 3, Article 15 (September 2012), 26 pages.

DOI $=10.1145 / 2355585.2355588$ http://doi.acm.org/10.1145/2355585.2355588

\section{INTRODUCTION}

The upwardly spiraling operating costs of the infrastructure for enterprise-scale computing demand efficient power management in server environments. It is difficult to achieve efficient power management in data centers, as they usually overprovision their power capacity to address the worst case scenarios. This calls for quantitatively

This article is an extension of earlier work presented at USENIX 2010 Workshop on Power-Aware Systems and Computing (Hotpower'10), entitled "Chaotic Attractor Prediction for Server Run-Time Energy Consumption."

This work was supported in part by the U.S. Department of Energy (DOE) under Award Number DE-FG0204ER46136 and by the Board of Regents, State of Louisiana, under Contract Number DOELEQSF(2004-07)ULL.

Authors' addresses: A.W. Lewis, Center for Advanced Computer Studies, University of Louisiana, Lafayette, LA 70504; email: awlewis@cacs.louisiana.edu. N.-F. Tzeng, Center for Advanced Computer Studies, University of Louisiana, Lafayette, LA 70504: email: tzeng@cacs.louisiana.edu.

Permission to make digital or hard copies of part or all of this work for personal or classroom use is granted without fee provided that copies are not made or distributed for profit or commercial advantage and that copies show this notice on the first page or initial screen of a display along with the full citation. Copyrights for components of this work owned by others than ACM must be honored. Abstracting with credit is permitted. To copy otherwise, to republish, to post on servers, to redistribute to lists, or to use any component of this work in other works requires prior specific permission and/or a fee. Permissions may be requested from Publications Dept., ACM, Inc., 2 Penn Plaza, Suite 701, New York, NY 10121-0701 USA, fax +1 (212) 869-0481, or permissions@acm.org.

(c) 2012 ACM 1544-3566/2012/09-ART15 $\$ 15.00$

DOI 10.1145/2355585.2355588 http://doi.acm.org/10.1145/2355585.2355588 
understanding the relationship between power consumption and thermal envelope at the system level so as to optimize the use of deployed power capacity in the data center.

Current AMD and Intel processors employ different techniques for processor-level power management [AMD 2008; Intel 2009]. In general, those techniques take advantage of the fact that reducing switching activity within the processor lowers energy consumption and that application performance can be adjusted to utilize otherwise idle time slacks on the processor for energy savings [Contreras and Martonosi 2005]. Power profiles of the Intel Pentium architecture have been studied for workstations [Isci and Martonosi 2003a, 2003b, 2006] and servers [Bircher et al. 2004; Bircher and John 2007; Lee and Skadron 2005]. Recent investigation into the power profiles of servers based on the NUMA architecture (for example, the AMD64 [AMD 2007], the Intel Nehalem [Intel 2009], and IBM POWER7 processors [Ware et al. 2010; Brochard et al. 2010]) indicates that managing server power profiles is complicated by the existence of multiple cores per processor [Kansal et al. 2010; Tsirogiannis et al. 2010; Lewis et al. 2010; McCullough et al. 2011]. As a result, this article presents an effective full-system continuous model with system-wide prediction of energy consumption for server blades built on multicore processors.

Our model treats a single server blade as one closed black-box system, relying on the fact that measured energy input into the system can be a function of the work done by the system (in executing its computational tasks) and of residual thermal energy given off by the system during execution. It utilizes the hardware performance counters (PeCs) of the server for relating power consumption to its consequent thermal envelope, enabling dynamical control of the thermal footprint under given workload. With PeCs, the computational load of a server can be estimated using relevant PeC readings together with a set of operating system's performance metrics. The selection of $\mathrm{PeCs}$ and metrics is guided by the observation that the computational load in processors that use a Non-Uniform Memory Access (NUMA) architecture can be estimated by the amount of data transferred to/from memory and among processors over system buses. The result is that the underlying system is a dynamic system whose behavior can be described as a system of deterministic differential equations whose solution can be estimated via time-series approximation. Our energy consumption prediction treats observed readings of available PeCs in a discrete time series and resorts to statistical approximation to compensate for those model components that cannot be determined exactly.

Generally, a time series model observes past outcomes of a physical phenomenon to anticipate future values of that phenomenon. Many time series-based models of processor energy consumption have been considered [Rivoire 2008; Bhattacharjee and Martonosi 2009; Powell et al. 2009; Reich et al. 2010; Bircher and John 2011], with recent work extending such models into the thermal domain [Coskun et al. 2008]. Time series are typically handled in three broad classes of mechanisms: auto-regressive, integrated, and moving average models [Box et al. 1994]. Each of these classes assumes a linear relationship between the dependent variable(s) and previous data points. However, energy consumption, ambient temperature, processor die temperatures, and CPU utilization in computers can be affected by more than just past values of those measurements made in isolation from each other [Bertran et al. 2010; McCullough et al. 2011]. Our analysis of experimental measurements of key processor PeC readings and performance metrics reveals that the measured readings and metrics of a server over the time series do not possess linearity and are chaotic in nature. This chaotic nature may result from the behavior of various server components, like DC-DC switching power converters commonly used by power circuitry in modern computers [Hamill et al. 1997; Tse and Di Bernardo 2002]. It thus leads to our development of a Chaotic 
Attractor Predictor (CAP) that models the dynamics of the underlying system of differential equations.

Servers based on the HyperTransport bus [HyperTransport Technology Consortium 2007] and the Intel QuickPath Links [Intel 2009] are chosen as representatives to validate our CAP for estimating runtime power consumption. CAP takes into account key thermal indicators (like ambient temperatures and die temperatures) and system performance metrics (like performance counters) for system energy consumption estimation within a given power and thermal envelope. It captures the chaotic dynamics of a server system without complex and time-consuming corrective steps usually required by linear prediction to account for the effects of nonlinear and chaotic behavior in the system, exhibiting polynomial time complexity. This work demonstrates that appropriate provision of additional PeCs beyond what are provided by a typical server is required to obtain more accurate prediction of system-wide energy consumption.

\section{PRIOR WORK}

Used to guide power management mechanisms in server systems, power models can be classified into two broad categories: simulation-based and analytical models. Although simulation may provide details and breakdowns of energy consumption, it is usually done statically in an off-line manner, is slow, and does not scale well nor apply favorably to realistic applications and large datasets. For example, the HotSpot thermal simulator [Skadron et al. 2004] models thermal behavior (and, indirectly, power behavior) by building a network of thermal resistances and capacitances to account for heating and power dissipation on a circuit. Such models must accurately reflect empirical data from the real systems being simulated. Studies of the power characteristics of the SPEC CPU 2000 and CPU 2006 benchmarks suites executed on the AMD Athlon 64 processor [Mesa-Martinez et al. 2007] and thermal measures [Mesa-Martinez et al. 2010] show that simulation-based models (1) need long execution times to accurately predict power behavior and (2) have problems dealing with key thermal metrics. Mercury [Heath et al. 2006] attempts to address these issues by adopting a hybrid approach with an out-ofband solver that processes component observations collected from a monitor daemon on the target system. The solver in this system uses collected information to simulate the thermal behavior in the system. The Mercury infrastructure was used to construct Freon and FreonEC for thermal and energy management of clusters. However, the statistical analysis of methods similar to those used in Freon indicate significant issues in this case [Davis et al. 2011]. Therefore, simulation-based models are unsuitable when dynamic power and thermal optimization are concerned [Economou et al. 2006].

Analytical models, by contrast, rely on power measurements at the constituent components of a server via sampling its hardware and software performance metrics, that is, processor PeCs (performance counters) and operating system performance metrics. PeCs are hardware registers that can be configured to count various micro-architectural events, such as branch mispredictions and cache misses. Attempts have been made to reconcile analytic models by mapping program phases to events [Isci and Martonosi 2006]. Common techniques for associating PeCs and/or performance metrics with energy consumption adopt linear regression to map collected metrics to energy consumed during program execution [Contreras and Martonosi 2005; Economou et al. 2006; Isci and Martonosi 2003b].

Models have been built for the processor, storage devices, single systems, and groups of systems in data centers [Kadayif et al. 2001; Isci and Martonosi 2003b]. For example, memory manufacturers provide clear architectural documentation concerning the power consumption of their products [Micron, Inc. 2007]; this information is regularly used as the foundation for modeling DRAM power usage [Liu et al. 2008; Lewis et al. 2008]. Similarly, detailed thermal and power models of storage devices [Gurumurthi 
et al. 2005] have been proposed. However, those models considered only the maximum internal data rate, thereby inadequate for our purpose of runtime measurement. At the processor level, Bellosa et al. [2003] and Contreras and Martonosi [2005] created power models that linearly correlated power consumption with PeCs. Those models are simple and fast with low overhead [Bircher and John 2007; Powell et al. 2009; Bircher and John 2011], but they are sensitive to benchmarks chosen for model calibration and often suffer from large worst-case errors, making them unsuitable for predictive purposes [McCullough et al. 2011]. Furthermore, they do not consider full-system power consumption.

The black-box approach, without detailed knowledge of underlying hardware, has been applied recently to full-system modeling [Bircher and John 2007; 2011] by using PeCs to estimate power consumption of $\mathrm{I} / \mathrm{O}$ devices, in addition to the processor. In general, full-system models can be created using operating system CPU utilization [Fan et al. 2007] and similar metrics [Heath et al. 2005] in addition to PeCs. Such full-system models, like MANTIS [Economou et al. 2006; Rivoire 2008] and trickle-down power modeling [Bircher and John 2011], relate usage information to the power of the entire system rather than of its individual components. Each of those models requires one or more calibration phases to determine the contribution of each system component to overall power consumption. The accuracy and the portability of full system power models were assessed earlier [Rivoire et al. 2008], revealing that reasonable accuracy across machines and different workloads was achievable by considering both $\mathrm{PeCs}$ (performance counters) and operating system performance metrics, since they together may capture all components of system dynamic power consumption.

Auto-regressive (AR) techniques have been adopted popularly to construct power and temperature models for servers [Coskun et al. 2008; Powell et al. 2009; Bircher and John 2011], since they are simple and efficient. The linear AR models attempt to correlate $\mathrm{PeCs}$ to system behavior, with an average error of five to twenty-five percent (depending on architecture and benchmarks). Unfortunately, their maximum errors tend to be ill-conditioned, because of their stationary nature. In a stationary process, the probability distribution does not change with time, nor do the mean and the variance. Hence, AR and auto-regressive/moving average (ARMA) models are not suited for data that exhibits sudden bursts of large amplitudes at irregular time epochs, due to their assumptions of normality [Tong 1993]. Given workload dynamics of a server vary in time and its power profiles often diverge over time, effort has been made to accommodate this diverse behavior [Mesa-Martinez et al. 2007; Coskun et al. 2008] so as to permit continuing use of AR and ARMA models. This way, however, negatively impacts the performance advantage resulting from auto-regressive techniques, namely, their simplicity.

Lately, the power consumption data collected during benchmark execution have signified their increasing nonlinearity over time. For example, an analysis of published results for the SPECpower_ssj2008 benchmark [Varsamopoulos et al. 2010; Hsu and Poole 2011] revealed maximum errors as large as $40 \%$ when modeling the benchmark results using linear two-point interpolation with the observation that the behavior of the power curves of this benchmark was neither linear nor convex. A variation of the MANTIS power model [Economou et al. 2006] was used to analyze the power curves of the SPEC CPU2006, PARSEC multithreaded benchmark suite [Bienia 2011], and a set of synthetic system stress benchmarks [McCullough et al. 2011]. This work found for multicore processors that the mean relative error doubled as compared to when the benchmark was restricted to executing on a single core. Furthermore, mean relative errors for individual subsystems, such as the CPU, were significantly higher, with an average error of $10-14 \%$ and the largest error as much as $150 \%$ for some workloads. 
The cause of nonlinear behavior in power data may be attributed to a number of factors. First, while the assumption of linearity depends upon homogeneous workloads, observations of the behavior of SPEC CPU benchmarks in both physical and virtual machine environments reveal that distributing execution tasks across multicore processors is rarely uniform to have homogeneous workloads [Kansal et al. 2010]. Second, modern processors have no simple features that can be abstracted easily to permit linear models (for example, effects such as cache contention, processor performance optimizations, and hidden device states) [McCullough et al. 2011].

Dealing with workload dynamics without high computational complexity requires efficient estimation able to address inherent nonlinearity in the time series. One approach follows local polynomial fitting via weighted least squares regression [Fan and Gijbels 1996] or its variation [Singh et al. 2009]. Another approach is to utilize a moving average technique for smoothing the data by computing an average of the subset of data from the time series observations. For example, an exponentially weighted moving average will weight the members of the subset in geometrically decreasing order to give less weight to samples as they are further away in time [NIST 2010]. A third approach to fitting nonlinear curves with varying degrees of smoothness in different locations makes use of the derivatives of an approximating function with discontinuities. This can be accomplished by employing splines with discontinuities existing at points identified as knots. An example of this approach is Multivariate Adaptive Regression Splines (MARS) [Friedman 1991], which models the time series as a weighted sum of basis functions $B_{i}(x)$ and constant coefficients $c_{i}$ :

$$
f(x)=\sum_{i=1}^{k} c_{i} B_{i}(x),
$$

where each of the basis functions can take the form of (1) a constant 1, with only one such term present, the intercept, (2) a hinge function in the form of $\max (0, x-c)$ or $\max (0, c-x)$, or $(3)$ a product of two or more hinge functions. MARS is suitable for modeling power behavior because of their good balance between bias and variance. A model with low bias signifies that it is flexible enough to address nonlinearity while sufficiently constrained to maintain low variance. More details about MARS can be found in the Appendix.

Chaotic systems are ubiquitous in nature, found in many different physical domains. One key feature of such a dynamic system is its sensitivity to initial conditions, whose small difference $\Delta x$ can result in the marked difference of $\Delta x e^{\lambda t}$ after time $t$, for a certain $\lambda>0$. This exponential separation signifies that even a small difference or error may lead to large divergence in the near future.

From a mathematical standpoint, chaos can be produced by both continuous and discrete systems. A continuous system expressed by a differential equation

$$
\frac{d x(t)}{d t}=F(x(t))
$$

with at least three degrees of freedom $x(t)=\left[x_{1}(t), x_{2}(t), x_{3}(t), \ldots, x_{m}(t)\right]$, can be related to a companion discrete system of

$$
x_{n+1}=f\left(x_{n}\right),
$$

by considering Equation (3) as a projection of the flow for Equation (2) on a surface. Three conditions are necessary for such a system to show chaos: (1) the differential equation and companion discrete system are deterministic, (2) the functions of $f$ and $F$ must be nonlinear, and (3) the discrete system must have a positive Lyapunov exponent [Liu 2010]. 


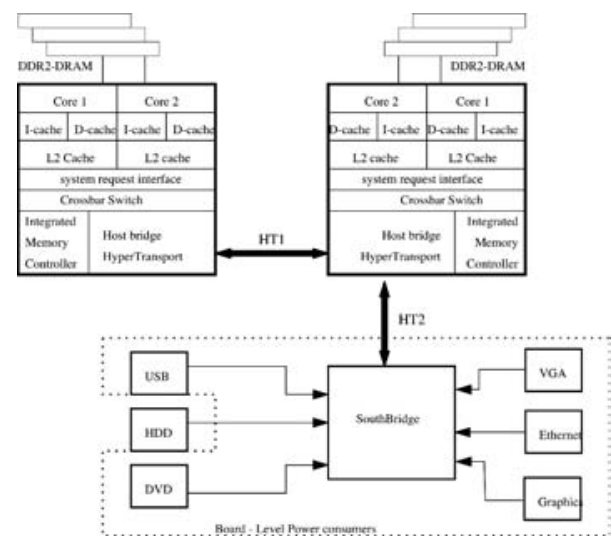

Fig. 1. AMD Opteron architecture.

\section{SYSTEM MODELING}

In order to develop a deterministic continuous energy consumption model based on computational load of the system, we consider $E_{d c}$, the total DC power input to the system, at the output of the power supply. Most servers operate on the AC input, with efficiency of power conversion from AC to DC equal to $72-80 \%$ (depending on the system load [Ton et al. 2008]) and with the DC output delivered in the domains of $+/-12 \mathrm{~V},+/-5 \mathrm{~V}$, and $+/-3.3 \mathrm{~V}$ [Server System Infrastructure Consortium 2004]. Typically, two 12 Vdc lines supply power to the processor's hard drive(s) and cooling fans in the system. The $5 \mathrm{Vdc}$ and $3.3 \mathrm{Vdc}$ lines are dedicated to supplying power to the support chips and peripherals on the board.

Energy delivered to a server system, $E_{d c}=E_{\text {system }}$, can be expressed as a sum of energy consumed by constituent sub-systems in the server. Generally, there are five sources of energy consumption in a server system:

$E_{\text {proc }}$ : energy consumed in the processor due to computation,

$E_{\text {mem }}$ : energy consumed by DRAM chips,

$E_{h d d}$ : energy consumed by the hard disk drive(s),

$E_{\text {board }}$ : energy consumed by peripherals in support of board the operations, including all devices in multiple voltage domains across the board (like chip-set chips, voltage regulators, bus control chips, connectors, interface devices, etc.), $E_{e m}$ : energy consumed by all electrical and electromechanical components in the server, including fans and other support components.

Total energy consumed by the system with a given computational workload can be expressed as:

$$
E_{\text {system }}=E_{\text {proc }}+E_{\text {mem }}+E_{\text {hdd }}+E_{\text {board }}+E_{\text {em }}
$$

Each of the above terms is explored in turn by following an energy conservation principle. In order to get a true measure of the computational load on the system, our approach snoops on bus transactions per unit time (indicated by $\mathrm{PeC}$ readings), measures the temperature changes (in die and ambient sensor readings), and records the speeds of cooling fans, in the course of job execution. The use of those PeCs and metric readings fits well to NUMA-based multicore processors.

\subsection{Processor Energy Consumption}

Consider the AMD Operton architecture and the Intel Nehalem architecture, as depicted in Figures 1 and 2. The former is a NUMA-based processor (Figure 1), with 


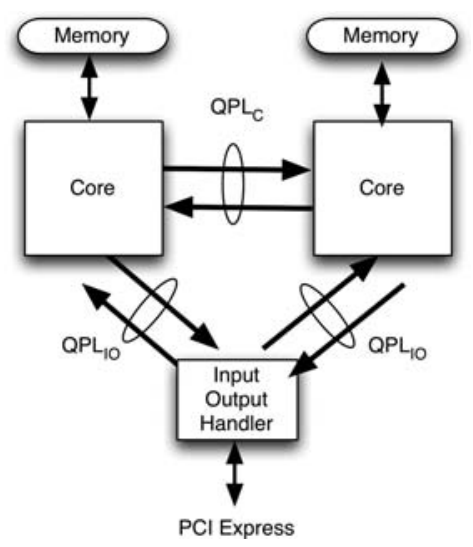

Fig. 2. Intel Xeon (Nehalem) architecture.

Northbridge functionality incorporated in the processor core and each core responsible for local access to the memory connected to that Northbridge logic (shown in Figure 1 as "Integrated Memory Controller"). Processor cores on a single die are connected via a crossbar to the HyperTransport bus (i.e., HT1) between processors. A coherent bus protocol is used to ensure memory consistency between processor cores on each die. In addition, the master processor in the system is connected via a second HyperTransport bus (i.e., HT2) to the Southbridge device that manages connections to the outside world. A similar structure exists in the Intel Xeon Nehalem architecture. Unlike the AMD Operton, each Nehalem processor is connected to an Input-Output handler, which provides the Southbridge with connecting functions for off-chip resources.

It is observed that work done by any of the processors, as the heart of energy consumption in a server system, can be quantified in terms of bus transactions in and out of the processors. Traffic on the external buses provides a measure of how much data is processed by the processor. In the case of the NUMA architecture, this quantity can be measured by determining the amount of data transmitted over interconnect between processor cores (HyperTransport for AMD processors, QPI links for recent Intel processors). Our energy consumption model aims to treat each processor as a black box, whose energy consumption is a function of its work load (as manifested by core die temperatures measured at the system level by ipmitool through sensors on the path of the outgoing airflow from the processor). In practice, when estimating processor power consumption based on PeCs (performance counters), there are only a limited number of PeCs for tools, like cpustat, to track simultaneously.

For the AMD dual-core Operton architecture (shown in Figure 1), traffic on the HT buses is viewed as a representative of the processor workload, reflecting the amount of data being processed by a processor (i.e., its involved cores). The HT2 bus is noncoherent and connects one of the two processors to the Southbridge (whereas the Northbridge is included on the Opteron processor die). Thus, traffic on the HT2 bus reveals hard-disk and network transactions. This model scales by considering the effect of network traffic and disk I/O transactions. HT1 is a coherent bus between the two SMP processors and, as such, PeCs on HT1 provide accurate estimation on the processing load of cores executing jobs. Per-core die temperature readings are directly affected by the number of transactions over the HT1 bus. A similar observation holds for the QPL links present in the Intel Nehalem architecture, with traffic between its two cores reflected by transactions on QuickPath Links between the cores, denoted by $Q P L_{C}$ (see Figure 2). 
Therefore, total processor power consumption at time $t, P_{\text {proc }}(t)$, is related to processor temperature readings and the estimated amount of data being processed at the time, and it can be expressed as a function of three metrics: die temperature readings for processors 0 and 1, and the number of bus transactions (i.e., traffic over HT1 for the AMD server and over $Q P L_{C}$ for the Intel server). We have processor energy consumption between times $t_{1}$ and $t_{2}$ as follows:

$$
E_{\text {proc }}=\int_{t_{1}}^{t_{2}}\left(P_{\text {proc }}(t)\right) d t
$$

\subsection{DRAM Energy Consumption}

Energy consumed by the DRAM banks is directly related to the number of DRAM $\mathrm{read} / \mathrm{write}$ operations involved during the time interval of interest, and the number is reflected by (1) the last-level cache misses for all $N$ constituent cores $\left(C M_{i}(t), i=\right.$ $1,2, \ldots, N)$ in the server when executing jobs and (2) the data amount due to disk accesses for OS support (like page tables, checkpoints, virtual environments) and due to performance improvement for peripheral devices (like buffered data for disks and optical devices, spooled printer pages). The data amount in (2), named $D B(t)$, is reflected by traffic over $\mathrm{HT}_{2}$ (or QuickPath links between the two cores and the Input/Output handler, denoted by $Q P L_{I O}$ ) for the AMD Opteron server (or the Intel Xeon server), as demonstrated in Figure 1 (or Figure 2). This is because network traffic does not exist in either testing server, which comprises only a single chip. Additional energy contributors include activation power and DRAM background power (due to leaking currents), represented by $P_{a b}$. As stated earlier [Micron, Inc. 2007], DRAM activation power and background power can be obtained from the DRAM documentation, and they together amount to $493 \mathrm{~mW}$ for one DRAM module in our AMD Opteron server. Consumed energy over the time interval between $t_{1}$ and $t_{2}$ can be expressed by

$$
E_{\text {mem }}=\int_{t_{1}}^{t_{2}}\left(\left(\sum_{i=1}^{N} C M_{i}(t)+D B(t)\right) \times P_{D R}+P_{a b}\right) d t
$$

where $P_{D R}$ refers to DRAM read/write power per unit data.

\subsection{Hard Disk Energy Consumption}

Energy consumed by the hard $\operatorname{disk}(\mathrm{s})$ is approximated by using a combination of relevant PeCs and drive ratings. Building a full-system model of disk drive energy consumption is complicated by the distance (from the processor) and the high latency of the disk drive. Two earlier modeling approaches exist, with one analyzing drive performance and capacity based on the physical characteristics of the drive [Gurumurthi et al. 2005] and the other utilizing PeCs (which measure DMA accesses on the memory bus, uncacheable accesses, and interrupts) to estimate the value of $E_{h d d}$ [Bircher and John 2011].

By contrast, our disk model is interested in the amount of information being transferred back and forth to the device. So, it is possible to calculate this value using information collected by the operating system and physical characteristics of the disk drive. Both our test servers use the Hitachi's SATA hard disk (whose specification and relevant power consumption figures are listed in Table I). Based on the physical, electrical, and electromechanical parameters of a hard disk, one can construct its detailed power consumption model. However, a cruder but simpler model can be obtained from the typical power consumption data of hard disks and pertinent PeCs, including (1) the number of reads and writes per second to the disk and (2) the amount of data (in kilobytes) read from and written to the disk. Those PeCs can be measured by the tool 
Table I. Hitachi HDT725025VLA360 Disk Power

Parameters

\begin{tabular}{ll}
\hline Parameter & \multicolumn{1}{c}{ Value } \\
\hline Interface & Serial ATA \\
Capacity & $250 \mathrm{~GB}$ \\
Rotational speed & $7200 \mathrm{rpm}$ \\
Power & \\
Spin up & $5.25 \mathrm{~W}$ (max) \\
Random read, write & $9.4 \mathrm{~W}$ (typical) \\
Silent read, write & $7 \mathrm{~W}$ (typical) \\
Idle & $5 \mathrm{~W}$ (typical) \\
Low RPM idle & $2.3 \mathrm{~W}$ (typical for $4500 \mathrm{RPM})$ \\
Standby & $0.8 \mathrm{~W}$ (typical) \\
Sleep & $0.6 \mathrm{~W}$ (typical) \\
\hline
\end{tabular}

of iostat, arriving at approximate disk power consumption, $E_{h d d}$, as:

$$
\begin{aligned}
E_{h d d}= & P_{\text {spin-up }} \times T_{\text {su }}+P_{\text {read }} \sum N_{r} \times T_{r} \\
& +P_{\text {write }} \sum N_{w} \times T_{w}+\sum P_{\text {idle }} \times T_{i d}
\end{aligned}
$$

where $P_{\text {spin-up }}$ is the power required to spin-up the disk from 0 to full rotation, and $T_{s u}$ is the time required to achieve spin up, typically about $10 \mathrm{sec}$. $P_{\text {read }}$ (or $P_{\text {write }}$ ) is the power consumed per kilobyte of data read from (or written to) the disk, whereas $N_{r}$ (or $N_{w}$ ) is the number of kilobytes of data reads (or data writes) in time-slice $T_{r}$ from (or to) the disk. The Hitachi disk achieves read operations at $1.5 \mathrm{Gbits} / \mathrm{s}$, when consuming $530 \mathrm{~mA}$ current at $+5 \mathrm{~V}$, thereby exhibiting approximately $13.3 \mu \mathrm{W} / \mathrm{Kbyte}$. Similarly, it is found to consume $6.67 \mu \mathrm{W} / \mathrm{Kbyte}$ for write operations. The numbers of $N_{r}$ and $N_{w}$ can be obtained using iostat according to the chosen time slice.

There are two idle states for the disk: idle and unloaded idle (when disk read/write heads are unloaded). The time to go from the unloaded idle state to the idle state is usually less than 1 second (smaller than the resolution of iostat). Thus, a history match count in the iostat statistics with zero reads and writes signifies the periods in which the disk is idle, permitting us to compute idle energy consumption accordingly. iostat readings for the durations of switching to different disk power states may be obtained with a more in-depth analysis, which is not considered in this work.

\subsection{Board Energy Consumption}

The quantity of $E_{\text {board }}$ represents energy consumption caused by the support chipsets, control logic, buses, signal links, etc., and it usually falls into the $3.3 \mathrm{~V}$ and $5 \mathrm{~V}$ power domains. Exhibiting little variation, $E_{\text {board }}$ can be difficult to measure, as chipset power is drawn from multiple domains. Hence, earlier models [Kansal et al. 2010; Bircher and John 2011] treated $E_{\text {board }}$ as a constant. In our case, however, this value is obtained using current probe-based measurements. The results are measured over an interval of interest, $t_{\text {interval }}$ and exclude the effects of processor, disks, fans, and optical devices. Note that introducing the current sensors (possibly taking up to 28 for a server [Server System Infrastructure Consortium 2004]) to the power lines on the board will provide instantaneous current readings for use in $E_{\text {board }}$.

Aggregated power consumption effects on the board may be captured using ambient temperature readings on the board. Such readings can be obtained using system management tools commonly found in server environments (such as IPMI), and they are included in the set of our PeCs for energy consumption estimation. 


\subsection{Electromechanical Energy Consumption}

A server always involves electromechanical energy consumption, $E_{e m}$, which is mainly due to the electromechanical functions related to system cooling. Multiple fans often exist in a server for cooling. Power drawn by the $i^{t h}$ fan at time $t$ can be given by the following equation:

$$
P_{\text {fan }}^{i}(t)=P_{\text {base }} \times\left(\frac{R P M_{\text {fan }}^{i}(t)}{R P M_{\text {base }}}\right)^{3},
$$

where $P_{b a s e}$ defines the base power consumption of the unloaded system when running only the base operating system and no application workload. The $P_{\text {base }}$ value is obtained experimentally by measuring the current drawn on the $+12 \mathrm{~V}$ and $+5 \mathrm{~V}$ lines, using a current probe and an oscilloscope. There is a current surge at system start, which is neglected. Under nominal conditions, the $+12 \mathrm{~V}$ line draws approximately $2.2 \mathrm{~A}$ to power both blower fans in the AMD testing server. Total electromechanical energy consumption over a given task execution period of $T_{p}$ equals:

$$
E_{\text {em }}=\int_{0}^{T_{p}}\left(\sum_{i=1}^{N} P_{f a n}^{i}(t)\right) d t .
$$

\section{EFFECTIVE PREDICTION}

The current generation of server systems lacks (1) the complete set of measurement and monitoring capabilities and (2) data flow state capture mechanisms required in order to formulate the parameters of an exact analytical model. For example, the system board DC and AC power consumption cannot be easily split in measurements or analyses, due to the presence of large numbers of voltage/current domains, each with multiple components. Therefore, effective prediction on future power consumption based on past power consumption readings/measurements (obtained from $\mathrm{PeCs}$ and performance metrics) is critical.

In Equation (4), $E_{\text {system }}$ signifies total energy consumed by the system for a given computational workload, equal to the sum of five terms: $E_{\text {proc }}, E_{\text {mem }}, E_{h d d}, E_{\text {board }}$, and $E_{\text {em }}$. Adopting Equation (4) for server energy consumption estimation, one needs to predict change in $E_{\text {system }}$ over the time interval of $(t, t+\Delta t)$. Such prediction, following a time series to make observations of the server system, based on PeCs and performance metrics, can be approximated by

$$
E_{\text {system }}=\hat{f}\left(E_{\text {proc }}, E_{\text {mem }}, E_{\text {hdd }}, E_{\text {board }}, E_{\text {em }}\right),
$$

where the involved parameters correspond to the five server energy contributors modeled in Sections 3.1 to 3.5.

\subsection{Performance Counters and Metrics}

In our prediction approach, fourteen (14) observable PeCs and accessible performance metrics (referred to as "measures" collectively for simplicity) are involved in the AMD server, as listed in Table II. They are grouped into five clusters, depending on their relevance to the server energy contributors. More specifically, the top three measures are related to $E_{\text {proc }}$, named $M P_{\text {proc }}^{A M D}=\left[T_{C_{0}}, T_{C_{1}}, H T_{1}\right]$. The next five measures dictate $E_{\text {mem }}$, denoted by $M P_{m e m}^{A M D}=\left[H T_{2}, C M_{0}, C M_{1}, C M_{2}, C M_{3}\right]$. Those $C M_{i}$ measures, capturing the total $\mathrm{L} 2$ cache miss counts due to Core $i$, are registered at PAPI_L2_TCM (being OpenSolaris generic events equivalent to the matching event in the Linux PAPI performance counter library [London et al. 2001]) and mapped to the AMD performance counters at 0x7E (as defined in [AMD 2006]). The following two measures are 
Table II. PeCs and Performance Metrics for AMD Opteron Server

\begin{tabular}{cl}
\hline Variable & \multicolumn{1}{c}{ Measurement } \\
\hline$T_{C_{0}}$ & CPU0 Die Temp \\
$T_{C_{1}}$ & CPU1 Die Temp \\
$H T_{1}$ & HT1 Bus X-Actions \\
$H T_{2}$ & HT2 Bus X-Actions \\
$C M_{0}$ & Last-level Cache Misses due to Core0 \\
$C M_{1}$ & Last-level Cache Misses due to Core1 \\
$C M_{2}$ & Last-level Cache Misses due to Core2 \\
$C M_{3}$ & Last-level Cache Misses due to Core3 \\
$D_{r}$ & Disk bytes read \\
$D_{w}$ & Disk bytes written \\
$T_{A_{0}}$ & Ambient Temp0 \\
$T_{A_{1}}$ & Ambient Temp1 \\
$F_{C}$ & CPU Cooling Fan Speed \\
$F_{M}$ & Memory Cooling Fan Speed \\
\hline
\end{tabular}

Table III. PeCs and Performance Metrics for Intel Nehalem Server

\begin{tabular}{cl}
\hline Variable & \multicolumn{1}{c}{ Measurement } \\
\hline$T_{C_{0}}$ & CPU0 Die Temp \\
$T_{C_{1}}$ & CPU1 Die Temp \\
$Q P L_{C}$ & Transactions on QPL between Cores \\
$Q P L_{I O}$ & Transactions on QPLs for IO Handler \\
$C M_{0}$ & Last-level Cache Misses due to Core0 \\
$C M_{1}$ & Last-level Cache Misses due to Core1 \\
$D_{r}$ & Disk bytes read \\
$D_{w}$ & Disk bytes written \\
$T_{A_{0}}$ & Ambient Temp0 \\
$T_{A_{1}}$ & Ambient Temp1 \\
$T_{A_{2}}$ & Ambient Temp2 \\
$F_{C}$ & Memory Cooling Fan Speed \\
$F_{M 2 a}$ & Memory Cooling Fan Speed 2a \\
$F_{M 2 b}$ & Memory Cooling Fan Speed 2a \\
$F_{M 3 a}$ & Memory Cooling Fan Speed 3a \\
$F_{M 3 b}$ & Memory Cooling Fan Speed 3b \\
$F_{M 4 a}$ & Memory Cooling Fan Speed 4a \\
$F_{M 4 b}$ & Memory Cooling Fan Speed 4b \\
$F_{M 5 a}$ & Memory Cooling Fan Speed 5a \\
$F_{M 5 b}$ & Memory Cooling Fan Speed 5b \\
\hline
\end{tabular}

pertinent to $E_{h d d}$, represented by $M P_{h d d}^{A M D}=\left[D_{r}, D_{w}\right]$, which refer to the total numbers of bytes in disk reads and disk writes, respectively, during a period of 5 seconds (in our experiments) for all I/O devices (which are limited to the disk only, since no network traffic nor optical disks exist in the two testing servers), as recorded by the system activity monitor. The next two measures are related to $E_{\text {board }}$, indicated by $M P_{\text {board }}^{A M D}=\left[T_{A_{0}}, T_{A_{1}}\right]$, which register the temperature readings of two board locations where temperature sensors are placed. Finally, the last two measures determine $E_{\text {em }}$, shown by $M P_{e m}^{A M D}=\left[F_{C}, F_{M}\right]$, which provide speed information of the CPU cooling fan and the memory cooling fan. Collectively, each observation at time $t$ includes the 14 measures of $M P^{A M D}(t)=\left[M P_{\text {proc }}^{A M D}, M P_{\text {mem }}^{A M D}, M P_{h d d}^{A M D}, M P_{b o a r d}^{A M D}, M P_{e m}^{A M D}\right]^{T}$.

On the other hand, the Intel Nehalem server involves twenty (20) measures, as listed in Table III. Again, they are classified into five groups, each associated with one server energy contributor. Notice that $Q P L_{C}$ and $Q P L_{I O}$ are relevant to QuickPath Links (depicted in Figure 2), and they are associated with $E_{\text {proc }}$ and $E_{\text {mem }}$, respectively. In practice, however, there is just one single $\mathrm{PeC}$ for holding aggregated $Q P L_{C}$ and 
$Q P L_{I O}$ together. Among those measures listed in Table III, the top three are pertinent to $E_{\text {proc }}$, comprising $M P_{\text {proc }}^{\text {Intel }}$. The next three measures determine $E_{\text {mem }}$, forming $M P_{\text {mem }}^{\text {Intel }}$. Those two $C M_{i}$ measures indicate the total L3 cache miss counts due to Core $i, i=0$ or 1 . The cache miss counts record the last-level cache (i.e., L3) misses for the Intel Xeon processor on which our testing Intel server is built. They are reflected by the OpenSolaris generic event, PAPI_L3_TCM (as detailed in Sun Microsystems [2008] and Intel [2009]). The next two measures are related to $E_{h d d}$ (and constitute $M P_{h d d}^{\text {Intel }}$ ), signifying the total numbers of bytes in disk reads and disk writes, respectively, during a period of 5 seconds. The subsequent three measures dictate $E_{\text {board }}$, obtained from 3 temperature sensors placed on the board for ambient temperature readings; they form $M P_{\text {board }}^{\text {Intel }}$. Finally, the last nine measures determine $E_{\text {em }}$, offering speed information of those nine memory cooling fans, to constitute $M P_{e m}^{\text {Intel }}$. As a result, each observation for the Intel server at time $t$ comprises the 19 measures of $M P^{\text {Intel }}(t)=\left[M P_{\text {proc }}^{\text {Intel }}, M P_{\text {mem }}^{\text {Intel }}, M P_{\text {hdd }}^{\text {Intel }}, M P_{\text {board }}^{\text {Intel }}, M P_{\text {em }}^{\text {Intel }}\right]^{T}$.

A common prediction approach follows the linear auto-regressive (AR) combination of observation measures to predict the quantities in Equation (7) [Lewis et al. 2008]. It yields $E_{\text {system }}$ by adding up $f_{c o}\left(M P_{c o}\right)$ for all server energy contributors, with each $f_{c o}$ (due to Contributor co) being a linear summation of its constituent measures, as detailed in Appendix. Such a linear AR approach has characteristics that make it unsuitable for modeling server systems. Consider the traces of actual power shown in Figures 7 and 8 for the SPEC CPU2006 zeusmp benchmark as executed on an AMD Opteron or Intel Nehalem server. We saw indications of (1) periodic behavior and (2) large swings in the power draw throughout the course of the benchmark run. Similar scenarios were observed for other benchmarks on the AMD Opteron server and the Intel Nehalem server under this work. Linear regression-based prediction for power draw can mispredict substantially (up to $44 \%$, as indicated in Table X). Thus, it is reasonably conjectured that nonlinear dynamics do exist in server systems. Given large swings in power draw usually occur to a typical server and cannot be completely attributed to noise, more accurate prediction than linear auto-regression and MARS [Friedman 1991] is indispensable.

\subsection{Chaotic Prediction}

The continuous system expressed in Equation (4) can be viewed as a multivariate differential equation in the time domain (energy being power used in a time period). The time series approximation of a system solution can be viewed as a projection of the flow of Equation (4) onto a surface [Liu 2010]. The projection is defined in a way that the behavior (i.e., energy consumption) of the dynamic system is reflected in our discrete approximation (i.e., our time series measures).

We performed an analysis on the data collected from our test systems to determine if the behavior of our time series can be attributed to some form of chaotic behavior. A chaotic process is one that is highly sensitive to a set of initial conditions. Small differences in those initial conditions yield widely diverging outcomes in such chaotic systems. In order to determine whether a process is chaotic, we must be able to show that (1) it demonstrates high sensitivity to initial conditions and topological mixing, and (2) its periodic orbits are dense [Sprott 2003]. After analyzing our experimental data, we believe that the power consumption of a server demonstrates chaotic behavior.

In order to evaluate a server's sensitivity to initial conditions, we consider the Lyapunov exponents of the time series data observed while running those benchmarks described in the previous section. The Lyapunov exponent quantifies the sensitivity of a system such that a positive Lyapunov exponent indicates that the 
Table IV. Indications of Chaotic Behavior in Power Time Series (AMD, Intel)

\begin{tabular}{ccc}
\hline & $\begin{array}{c}\text { Hurst } \\
\text { Parameter } \\
(H)\end{array}$ & $\begin{array}{c}\text { Average } \\
\text { Lyapunov } \\
\text { Exponent }\end{array}$ \\
\hline bzip2 & $(0.96,0.93)$ & $(0.28,0.35)$ \\
cactusADM & $(0.95,0.97)$ & $(0.01,0.04)$ \\
gromacs & $(0.94,0.95)$ & $(0.02,0.03)$ \\
leslie3d & $(0.93,0.94)$ & $(0.05,0.11)$ \\
omnetpp & $(0.96,0.97)$ & $(0.05,0.06)$ \\
perlbench & $(0.98,0.95)$ & $(0.06,0.04)$ \\
\hline
\end{tabular}

system is chaotic [Sprott 2003]. The average Lyapunov exponent can be calculated using $\lambda=\lim _{N \rightarrow \infty} \frac{1}{N} \sum_{n=0}^{N-1} \ln \left|f^{\prime}\left(X_{n}\right)\right|$.

We found a positive Lyapunov exponent when performing this calculation on our dataset, ranging from 0.01 to 0.28 (or 0.03 to 0.35 ) on the AMD (or Intel) test server, as listed in Table IV, where each pair indicates the parameter value of the AMD server followed by that of the Intel server. Therefore, our data has met the first and the most significant criterion to qualify as a chaotic process.

The second indication of the chaotic behavior of the time series in Equation (7) is an estimate of the Hurst parameter $H$ for the datasets collected in each benchmark. A real number in the range of $(0,1)$, the Hurst parameter is in the exponents of the covariance equation for Fractional Brown motion (fBm) [Sprott 2003]. If the value of the Hurst parameter is greater than 0.5 , an increment in the random process is positively correlated and long range dependence exists in the case of time series. In a chaotic system, a value of $H$ approaching 1.0 indicates the presence of self-similarity in the system. As demonstrated in Table IV, the time series data collected in our experiments all have values of $H$ close to 1.0, ranging from 0.93 to 0.98 (or 0.93 to 0.97 ) on the AMD (or Intel) test server.

From a predictive standpoint, the unpredictable deterministic behavior of chaotic time series means that it is difficult to build a predictor that takes a global parametric view of the data in the series. However, it is possible to generate a highly accurate short-term prediction by reconstructing the attractor in the phase space of the time series and applying a form of least square prediction to the resulting vector space [Itoh 1995; Su 2010].

4.2.1. Chaotic Attractor Predictors. With the time series introduced in Equation (7), let $y_{t}$ be the value of $E_{\text {system }}$ at time $t, r$ be the total number of PeCs and performance measures to provide metric readings, and $X_{t}$ be the vector of those $r$ metric readings at time $t$. According to Taken's Delay Embedding Theorem [Sprott 2003], there exists a function $\hat{f}\left(X_{t}\right)$ whose behavior in the phase space reflects the behavior of the attractors in the original time series values $y_{t}$. Consequently, for given $\hat{f}$, a known $X_{t}$ reveals system energy consumption at time $t$, namely, $y_{t}$. If $X_{t}$ can be predicted accurately for future time $t$ (likely based on past metric readings), system energy consumption at future $t$ can be estimated properly. To this end, it is necessary to find a means for approximating $\hat{f}$.

We introduce the concept of Chaotic Attractor Prediction (CAP) that defines $\hat{f}$ in terms of least squares regression of a multivariate local polynomial of degree $r$. Multivariate local regression is a common nonparametric technique for time series approximations. With CAP, we extend this concept to predict the behavior of a chaotic time series by following the approximation method to take advantage of its polynomial time complexity while capturing the behavior dynamics of testing systems. 
Let $X$ be an observation (involving $r$ measures of

$$
M P(t+\Delta t)=\left[M P_{\text {proc }}, M P_{\text {mem }}, M P_{h d d}, M P_{\text {board }}, M P_{e m}\right]^{T}
$$

for a given server, as described earlier) at some future time $t+\Delta t$ and $X_{u}$ be a prior observation (involving $r$ metric readings of $M P(u)$ ) at time $u$ for $u=t-1, t-2, \ldots, t-p$. CAP localizes and addresses the possibility of noise in our observations through kernel weighting. This process starts with the standard multivariate normal density function of $K(x)=(2 \pi)^{-\frac{m}{2}} \exp \left(-\|X\|^{2} / 2\right)$ (where $\|X\|$ is the norm of vector $X$ ) for smoothing out values of a local neighborhood, over which our CAP is defined. Let the bandwidth of $\beta$ be a nonnegative number and $K_{\beta}(X)$ equal $K(X / \beta) / \beta$ [Fan and Gijbels 1996]. The function of $K_{\beta}$ serves as a kernel to smooth out noise in our original observations in a nonparametric manner. It has been shown that $\beta$ determines the degree of smoothing produced by the kernel [Fan and Yao 2005]. Selection of a small $\beta$ value does not adequately address issues of noise, while a too large $\beta$ value results in excessive bias in the results and may hide important dynamics of the underlying function $\hat{f}$ [Turlach 1993]. A preferred choice for $\beta$ can be computed by: $\beta=\left(\frac{4}{3 p}\right)^{\frac{1}{5}} \sigma$, where $\sigma$ is the standard deviation of observed values, estimated via the formula of $\bar{\sigma}=\operatorname{median}\left(\left|x_{i}-\bar{\mu}\right|\right) / 0.6745$, with $\bar{\mu}$ being the median of observed values [Bowman and Azzalini 1997].

An approximation for $\hat{f}$ is defined subsequently in terms of a locally weighted average [Box et al. 1994; Fan and Gijbels 1996] over the next $n$ observations, based on the prior $p$ observations of $X_{t-1}, \ldots, X_{u}, \ldots, X_{t-p}$ (each with $r$ measures, namely, $M P(u)=$ $\left[M P_{\text {proc }}, M P_{\text {mem }}, M P_{h d d}, M P_{\text {board }}, M P_{\text {em }}\right]^{T}$, as described earlier):

$$
\hat{f}(X)=\frac{\sum_{d=t}^{t+n-1} O_{p} * K_{\beta}\left(X_{d}-X\right)}{\sum_{d=t}^{t+n-1} K_{\beta}\left(X_{d}-X\right)}
$$

with $O_{p}=\left(X_{t-1}, X_{t-2}, \ldots, X_{t-p}\right)$.

The process can be improved by defining a local approximation via applying a truncated Taylor series expansion of $\hat{f}(X)$ for $X$ at nearby $x$ :

$$
\hat{f}(X)=\hat{f}(x)+\hat{f}^{\prime}(x)^{T}(X-x) .
$$

The coefficients of the polynomial $\hat{f}$ are then determined by minimizing

$$
\sum_{d=t}^{t+n-1}\left(X_{d}-a-b^{T}\left(X_{d}-x\right)\right)^{2} * K_{\beta}\left(X_{d}-x\right) .
$$

with respect to $a$ and $b$, which are estimators to $\hat{f}(x)$ and $\hat{f}^{\prime}(x)$, respectively. The predictor generated by solving Equation (8) can be explicitly written, according to [Box et al. 1994], as

$$
\hat{f}(x)=\frac{1}{n} \sum_{d=t}^{t+n-1}\left(s_{2}-s_{1} *\left(x-X_{d}\right)\right)^{2} * K_{\beta}\left(\left(x-X_{d}\right) / \beta\right)
$$

with $s_{i}=\frac{1}{n} \sum_{d=t}^{t+n-1}\left(x-X_{d}\right)^{i} * K_{\beta}\left(\left(x-X_{d}\right) / \beta\right)$, for $i=1$ or 2 . 
Table V. SPEC CPU2006 Benchmarks Used for Model Calibration

\begin{tabular}{|c|c|c|}
\hline \multicolumn{3}{|c|}{ Integer Benchmarks } \\
\hline bzip2 & $\mathrm{C}$ & Compression \\
\hline mcf & $\mathrm{C}$ & Combinatorial Optimization \\
\hline omnetpp & $\mathrm{C}++$ & Discrete Event Simulation \\
\hline \multicolumn{3}{|c|}{ FP Benchmarks } \\
\hline gromacs & C/F90 & Biochemistry/Molecular Dynamics \\
\hline cactusADM & C/F90 & Physics/General Relativity \\
\hline leslie3d & F90 & Fluid Dynamics \\
\hline $\mathrm{lbm}$ & $\mathrm{C}$ & Fluid Dynamics \\
\hline
\end{tabular}

4.2.2. CAP Creation. There are three steps involved in the process of creating a CAP predictor: (1) creating a training set for the process, (2) using the observations from the training set to find the appropriate delay embedding using Takens Theorem and then apply the nearest neighbors algorithm in the embedded set to identify the attractors, and (3) solving the resulting linear least squares problem that arises from applying Equation (8) to the attractors using the function expressed by Equation (9).

The training set for the predictor is constructed from a consolidated time series created by executing the SPEC CPU2006 [Henning 2006] benchmarks listed in Table V on target systems. The benchmarks were selected using two criteria: sufficient coverage of the functional units in the processor and reasonable applicability to the problem space. Components of the processor affect the thermal envelope in different ways [Kumar et al. 2008]. This issue is addressed by balancing the benchmark selection between integer and floating point benchmarks in the SPEC CPU2006 benchmark suite. Second, the benchmarks were selected from the suite based upon fit into the problem space. Each benchmark represents an application typical of the problems solved on high-performance application servers.

Each benchmark in the calibration set was executed with a sampling interval of $t=5$ seconds. The observations from each time interval were consolidated on this time interval using two methods: arithmetic mean (average) and geometric mean. Trial models were constructed using each method and a statistical analysis of variance indicated that time series generated from the geometric mean produced the best fit to the collected data.

4.2.3. Time Complexity. The time complexity of creating a predictor is governed by the third step in the process. The task of reconstructing the state space by delay embedding is linear in time as one must make up to $d$ passes through the observations, under the embedding dimension of $d$. Thus, the time required is $O(d n)$, where $n$ is the number of future observations. Then, it becomes a matter of applying a naive form of $k^{\text {th }}$ nearest neighbors algorithm to identify the points in the attractors. This step involves finding the squared distance of all the points in the nearest analogs in the Takens set and then sorting the result to determine the $d$-nearest neighbors. This step takes $O(n \log n+n)$. The high cost of computing the linear least squares solution in the third step is avoided by using the explicit expression given in Equation (9). The time complexity of computing this expression can be shown to be $O(n * n)$, with $O(n)$ due to computing $s_{i}$, for $i=1$ or 2. As a result, the time complexity for establishing a CAP predictor equals $O\left(n^{2}\right)$. It should be noted that the construction of a CAP predictor is done only once for a given server, irrespective of applications executed on the server. Such construction is based on past PeC observations (totally, $p$ of them) to predict the future PeC readings. As $p$ grows (with more past $\mathrm{PeC}$ observations involved), the time complexity of CAP increases linearly, as can be obtained in Equation (8). The actual computation time results under different $n$ and $p$ values for our CAP code implemented using MATLAB are provided in the next section. 


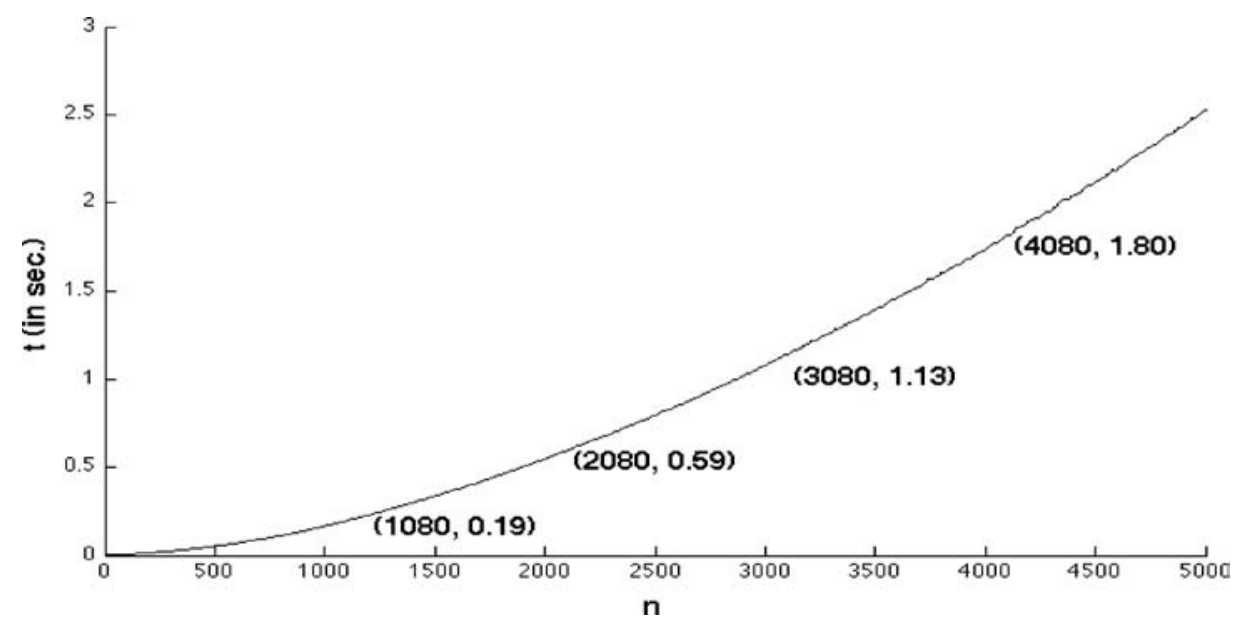

Fig. 3. CAP time complexity versus no. of future observations.

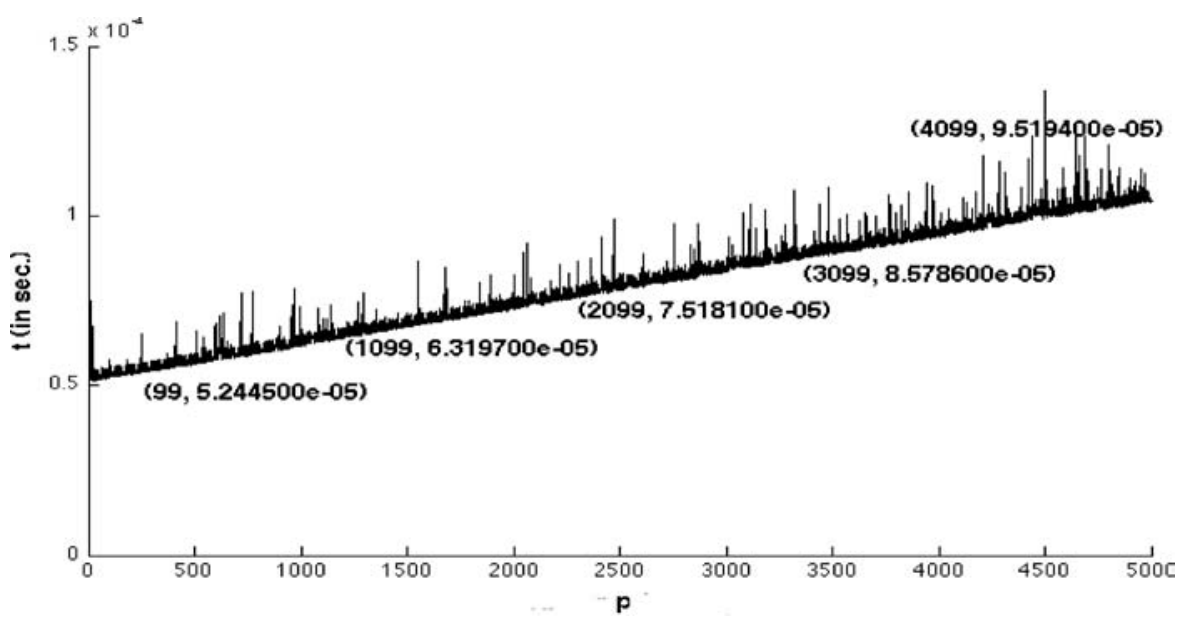

Fig. 4. CAP time complexity versus no. of past observations.

\section{EVALUATION AND RESULTS}

Experiments were carried out to evaluate the performance of the CAP power models built for approximating dynamic system solutions. The first experiment aimed to confirm the time complexity of CAP. Making use of MATLAB, our CAP code was executed on the two servers specified in Table VI. As the execution times on both servers provide the same trend, only those collected from the SUN Fire 2200 server (AMD Opteron) are demonstrated here. The code execution time results versus $n$ (the number of future observations) is illustrated in Figure 3, where the result curve confirms CAP time complexity in $O\left(n^{2}\right)$. Separately, the CAP execution time as a function of $p$ (the number of past observations) on the SUN Fire server is shown in Figure 4, where the time indeed is linear with respect to $p$, as claimed earlier in our time complexity subsection. In practice, a moderate $p$ (of, say, 100) and a small $n$ (of, say, 5) may be chosen under CAP for high accuracy and very low time complexity in real-time applications. The results of distant future (corresponding to a larger $n$ ) can be predicted by a stepwise process, with each step predicting the near future outcomes (using $n=5$ ). 


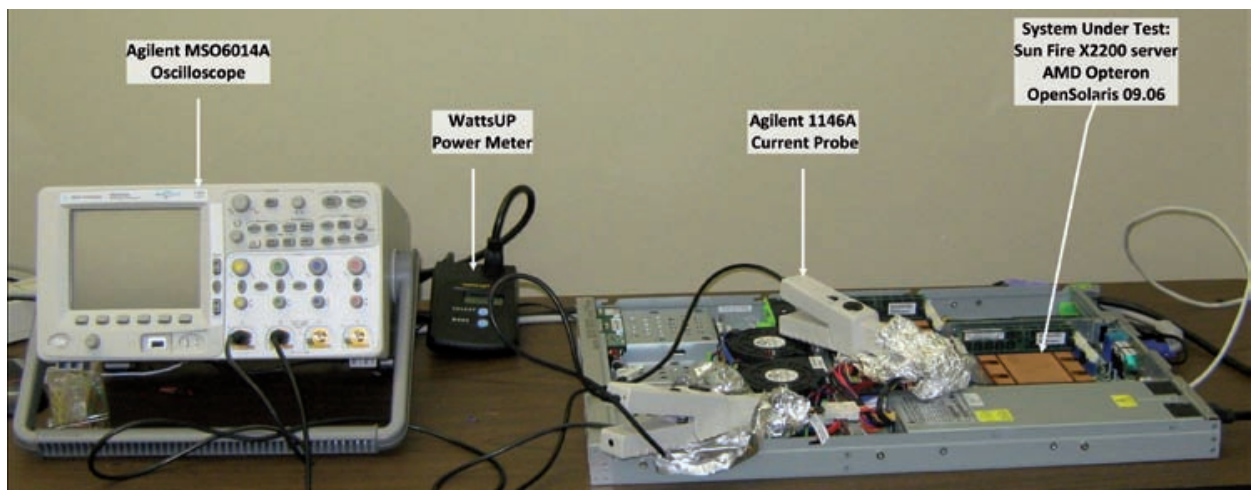

Fig. 5. Hardware test setup.

Table VI. Server Configurations for Evaluation

\begin{tabular}{lll}
\hline & Sun Fire 2200 & Dell PowerEdge R610 \\
\hline CPU & 2 AMD Opteron & 2 Intel Xeon (Nehalem) 5500 \\
CPU L2 cache & $2 \times 2 \mathrm{MB}$ & $4 \mathrm{MB}$ \\
Memory & 8GB & $9 \mathrm{~GB}$ \\
Internal disk & $2060 \mathrm{~GB}$ & $500 \mathrm{~GB}$ \\
Network & $2 \times 1000 \mathrm{Mbps}$ & $1 \times 1000 \mathrm{Mbps}$ \\
Video & On-board & NVIDA Quadro FX4600 \\
Height & 1 rack unit & 1 rack unit \\
\hline
\end{tabular}

Table VII. SPEC CPU2006 benchmarks used for evaluation

\begin{tabular}{ccl}
\hline \multicolumn{3}{c}{ Integer Benchmark } \\
\hline Astar & C++ & Path Finding \\
Gobmk & C & Artificial Intelligence: Go \\
FP Benchmarks & \\
\hline Gamess & F90 & Quantum chemical computations \\
Zeusmp & F90 & Computational Fluid Dynamics \\
\hline
\end{tabular}

\subsection{Evaluation Environment}

The operating system used in our test servers is OpenSolaris (namely, Solaris 11). Evaluation results are collected from the system baseboard controller using the IPMI interface via the OpenSolaris ipmitool utility. Processor performance counters are collected on a system-wide basis by means of cpustat, iostat, and ipmitool utilities in OpenSolaris. Of these, iostat and ipmitool are available across all UNIX-based operating systems commonly employed by data centers, while cpustat is an OpenSolaris-specific utility (which is being ported to Linux).

Four benchmarks from the SPEC CPU2006 benchmark suite were used for the evaluation purpose, as listed in Table VII), and they are different from those employed earlier for CAP creation (as listed in Table V). It is noted that selection of benchmarks for both calibration and evaluation were selected to sufficiently exercise processor, cache, and memory again per the decision criteria in Phansalkar et al. [2007], with the additional criterion of selecting workloads more common to server environments. As illustrated in Table VIII (per results from Phansalkar et al. [2007]), the mix of instructions exercises the key components of processor, memory, and cache in the dynamic system that we model with CAP. In addition, the four benchmarks used in our evaluation represent the type of high-utilization workloads in server environments per typical industry use of the SPEC CPU2006 benchmark suite [Cisco Systems 2010]. 
Table VIII. Branch and Memory Access Patters of Evaluation

Benchmarks

\begin{tabular}{lllll}
\hline Benchmark & $\begin{array}{l}\text { Inst Count } \\
\text { (Billions) }\end{array}$ & Branches & Loads & Stores \\
\hline astar & 1,200 & $15.57 \%$ & $40.34 \%$ & $13.75 \%$ \\
gamess & 5,189 & $7.45 \%$ & $45.87 \%$ & $12.98 \%$ \\
gobmk & 1,603 & $19.51 \%$ & $29.72 \%$ & $15.25 \%$ \\
zeusmp & 1,566 & $4.05 \%$ & $36.22 \%$ & $11.98 \%$ \\
\hline
\end{tabular}

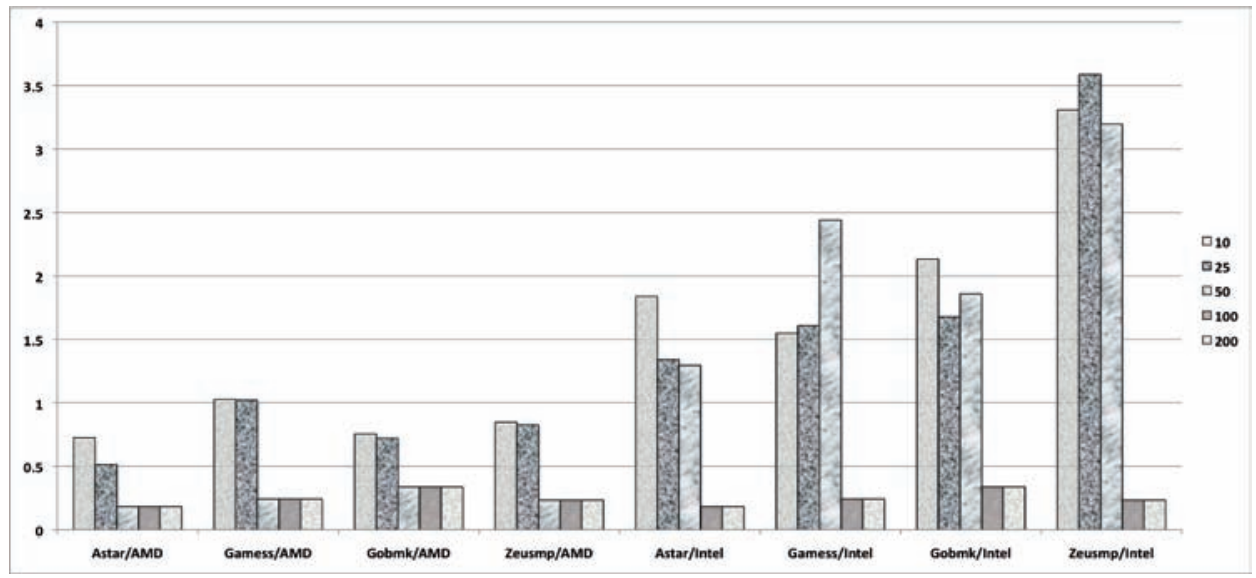

Fig. 6. Root Mean Square Error (RMSE) for different values of $p$

The benchmarks were executed on the two servers specified in Table VI, with performance metrics gathered during the course of execution. Power consumed is measured by a WattsUP power meter [Electronic Educational Devices, Inc. 2006], connected between the AC Main and the server under test (SUT). The power meter measures the total and average wattage, voltage, and amperage over the run of a workload. The internal memory of the power meter is cleared at the start of each run and the measures collected during the runs are downloaded (after execution completion) from meter's internal memory into a spreadsheet. Current flow on different voltage domains in the server is measured using an Agilent MSO6014A oscilloscope, with one Agilent 1146A current probe per server power domain $(12 \mathrm{v}, 5 \mathrm{v}$, and $3.3 \mathrm{v})$. This data was collected from the oscilloscope at the end of each benchmark execution on a server and then stored in a spreadsheet on the test host. The apparatus is shown in Figure 5.

\subsection{Results}

While the number of measures per observation $(r)$ is fixed for a given server in our evaluation (equal to 14 for the Sun Fire server and to 19 for Dell PowerEdge server), the CAP prediction time and accuracy depend on $p$ (the number of past observations) and $n$ (the number of future observations), as stated in Section 4.2.2. In our evaluation, the CAP prediction error rates of various benchmark codes for a range of $p$ under a given $n$ were gathered, as demonstrated in Figure 6 , where $n$ equals 5 . It can be seen from the figure that the error rates are fairly small (and stay almost unchanged) when $p$ is within 100 to 200, but they rise considerably when $p$ drops to 50 or below. In subsequent figures, the prediction results of CAP include only those for $n=5$ and $p=100$. Each benchmark was executed to collect the first $p=100$ points on the attractor, at which point the next $n=5$ points were used to compute the estimated power for the $t, t+1, \ldots, t+5$ energy estimates in each time cycle. 


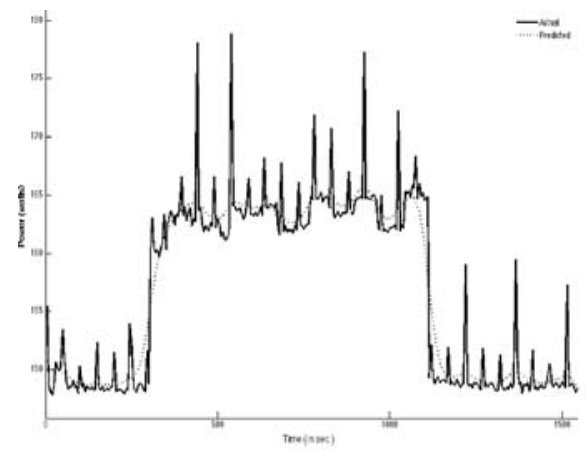

(a) Astar/CAP.

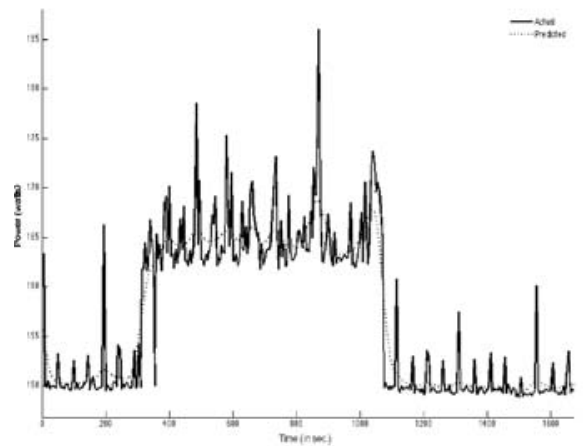

(c) Zeusmp/CAP.

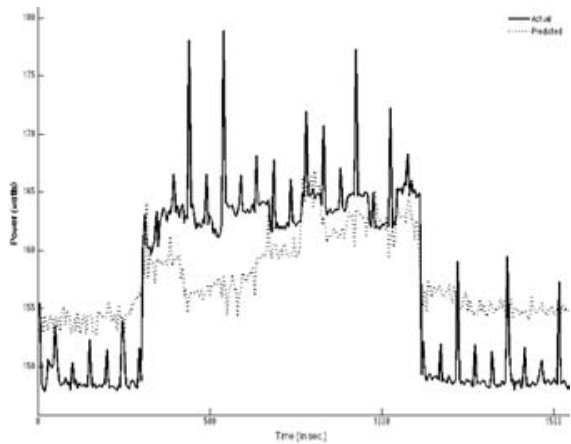

(b) Astar/AR(1)).

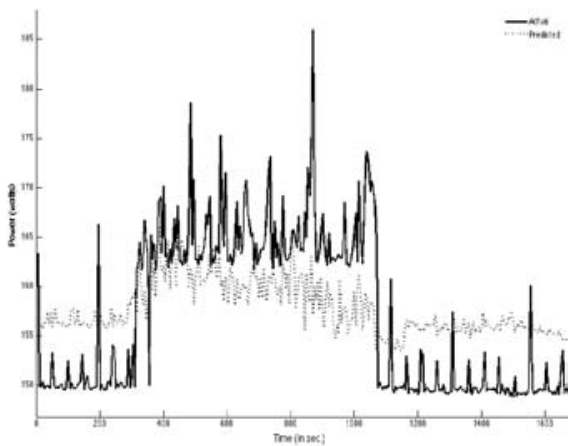

(d) Zeusmp/AR(1).

Fig. 7. Actual power results versus predicted results for AMD Opteron.

The predicted power consumption results of CAP during the execution of Astar and Zeusmp on a HyperTransport-based server are demonstrated in Figures 7(a) and 7(c). The predicted values are seen to track closely to the measured readings (obtained using the WattsUP power meter and indicated by solid curves), with the error rate ranging between $0.9 \%$ and $1.6 \%$. For comparison, the predicted power consumption outcomes during the execution of same selected benchmarks under AR(1) are depicted in Figures 7(b) and 7(d), where details of AR(1) can be found in Appendix. As expected, $\mathrm{AR}(1)$ exhibits poor outcomes over any given short execution window, with maximum errors ranging from $7.9 \%$ to $9.3 \%$, despite that the prediction error over the whole execution period may be less. CAP enjoys much better prediction behavior than its linear regressive counterpart.

The predicted power consumption results under CAP over the benchmark execution period for the QPL-based server (Dell PowerEdge) are demonstrated in Figures 8(a) and 8(c), where the actual power consumption amounts obtained by the WattsUP meter are shown by solid curves. Again, CAP is seen to exhibit impressive performance, tracking the actual amounts closely, with the error rate ranging between $1.0 \%$ and $3.3 \%$. The root mean square errors for CAP remain within small values. In contrast, $A R(1)$ suffers from poor prediction behavior, as can be discovered in Figures 8(b) and 8(d), where outcomes of same benchmarks executed on the Dell PowerEdge server are depicted. It yields the maximum error up to $20.8 \%$ (or 20.6\%) for the Astar (or Zeusmp) benchmark. 


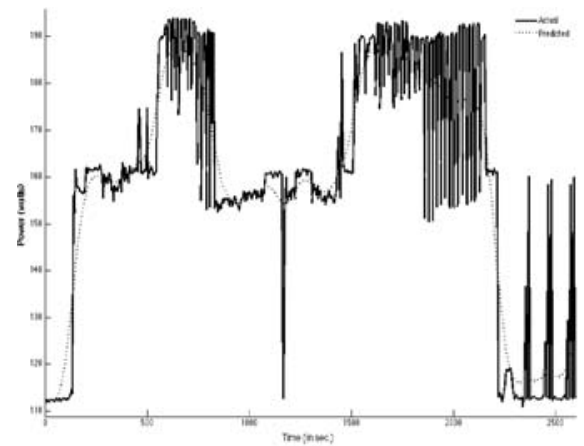

(a) Astar/CAP.

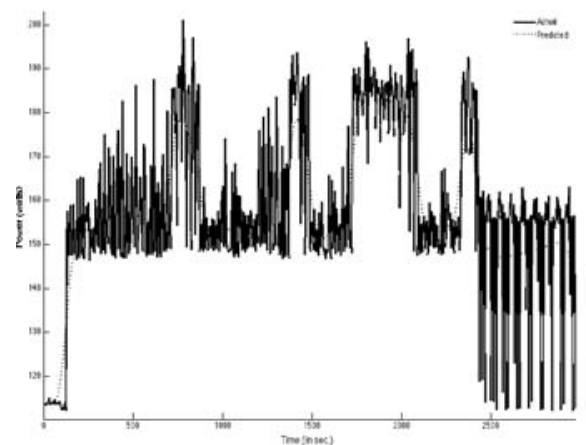

(c) Zeusmp/CAP.

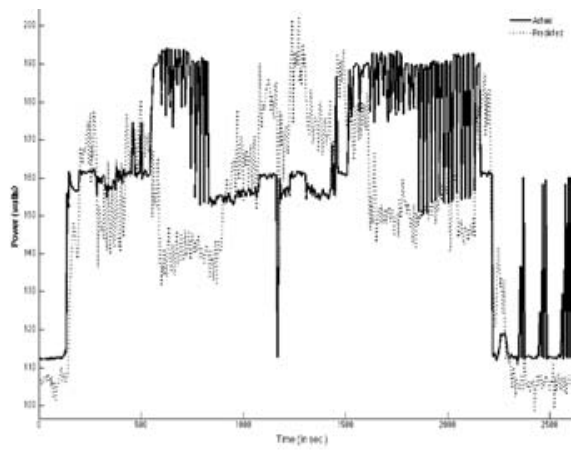

(b) Astar/AR(1)).

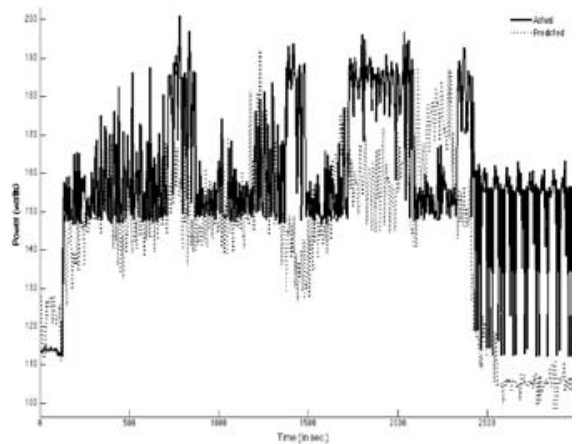

(d) Zeusmp/AR(1).

Fig. 8. Actual power results versus predicted results for an Intel Nehalem server.

Table IX. Model errors for CAP (under $n=5, p=100, r=14$ ), $A R(1), M A R S$, and EWMA on AMD Opteron server

\begin{tabular}{c|ccc|ccc}
\hline & \multicolumn{3}{|c|}{ CAP } & \multicolumn{3}{c}{ AR } \\
\hline & Avg & Max & Avg & Max \\
Benchmark & Err \% & Err \% & RMSE & Err \% & Err \% & RMSE \\
\hline Astar & $0.9 \%$ & $5.5 \%$ & 0.72 & $3.1 \%$ & $8.9 \%$ & 2.26 \\
Gamess & $1.0 \%$ & $6.8 \%$ & 2.06 & $2.2 \%$ & $9.3 \%$ & 2.06 \\
Gobmk & $1.6 \%$ & $5.9 \%$ & 2.30 & $1.7 \%$ & $9.0 \%$ & 2.30 \\
Zeusmp & $1.0 \%$ & $5.6 \%$ & 2.14 & $2.8 \%$ & $8.1 \%$ & 2.14 \\
\hline & \multicolumn{3}{|c|}{ MARS } & \multicolumn{3}{c}{ EWMA } \\
\hline & Avg & Max & & Avg & Max \\
Benchmark & Err \% & Err \% & RMSE & Err \% & Err \% & RMSE \\
\hline Astar & $2.5 \%$ & $9.3 \%$ & 2.12 & $1.2 \%$ & $7.9 \%$ & 2.40 \\
Gamess & $3.0 \%$ & $9.7 \%$ & 2.44 & $1.1 \%$ & $7.8 \%$ & 1.42 \\
Gobmk & $3.0 \%$ & $9.1 \%$ & 2.36 & $1.0 \%$ & $6.9 \%$ & 2.30 \\
Zeusmp & $2.8 \%$ & $7.9 \%$ & 2.34 & $1.8 \%$ & $9.2 \%$ & 2.01 \\
\hline
\end{tabular}

\subsection{Further Discussion}

Table IX (or X) compares the errors of evaluation benchmarks for the server with the HyperTransport (or QPL) structure, under four different prediction mechanisms: CAP, AR, MARS, and EWMA. Details of AR, MARS, and EWMA predictors can be found in Appendix. Large errors exhibited by AR, MARS, and EWMA overwhelm the 
Table X.

Model errors for CAP (under $n=5, p=100, r=19$ ), AR, MARS, and EWMA predictors on Intel Nehalem server

\begin{tabular}{c|ccc|ccc}
\hline & \multicolumn{3}{|c|}{ CAP } & \multicolumn{3}{c}{ AR } \\
\hline Benchmark & Avg & Max & Avg & Max \\
& Err \% & Err \% & RMSE & Err \% & Err \% & RMSE \\
\hline Astar & $1.1 \%$ & $20.8 \%$ & 1.83 & $5.9 \%$ & $28.5 \%$ & 4.94 \\
Gamess & $1.0 \%$ & $14.8 \%$ & 1.54 & $5.6 \%$ & $44.3 \%$ & 5.54 \\
Gobmk & $1.0 \%$ & $21.5 \%$ & 2.13 & $5.3 \%$ & $27.8 \%$ & 4.83 \\
Zeusmp & $3.3 \%$ & $20.6 \%$ & 3.31 & $7.7 \%$ & $31.8 \%$ & 7.24 \\
\hline & \multicolumn{3}{|c|}{ MARS } & & \multicolumn{3}{c}{ EWMA } \\
\hline & Avg & Max & & Avg & Max \\
Benchmark & Err \% & Err \% & RMSE & Err \% & Err \% & RMSE \\
\hline Astar & $5.4 \%$ & $28.0 \%$ & 4.97 & $3.7 \%$ & $32.4 \%$ & 2.98 \\
Gamess & $4.7 \%$ & $33.0 \%$ & 4.58 & $1.8 \%$ & $27.3 \%$ & 2.19 \\
Gobmk & $4.1 \%$ & $27.9 \%$ & 4.73 & $3.9 \%$ & $28.4 \%$ & 2.73 \\
Zeusmp & $11.6 \%$ & $32.2 \%$ & 8.91 & $5.0 \%$ & $31.3 \%$ & 2.81 \\
\hline
\end{tabular}

advantages gained from their simplicity. The table results indicate the limitations entailed by using a linear technique, such as AR time series, to predict dynamic system behavior; similar issues exist for piecewise and moving average techniques, such as MARS and EWMA. Earlier attempts were made to address this issue by incorporating corrective mechanisms in combination with these predictors. An example attempt employed machine learning to monitor misprediction, with recalibration invoked when required [Coskun et al. 2008]. CAP eliminates the need for any corrective mechanism by directly addressing the system dynamics, thereby avoiding wide drifts in prediction experienced by other prediction techniques.

The model developed in this article is valid for any dual-core/dual-processor system using NUMA memory access connected in a point-to-point manner using the HyperTransport or the QPL structures. However, it can be scaled to quad-core dual processors based on those two structures. One would expect to see a slight difference or variation in power prediction due to a greater or less affect of die temperatures on the other performance measures. Under a dual-core quad-processor server, for example, additional regression variables would be incorporated in $E_{\text {proc }}$, giving rise to more performance measures (i.e., a larger $r$ ). Similarly, more PeCs related to cache misses would then be involved in $E_{\text {mem }}$. The solution approach of CAP remains exactly identical, except for a larger $r$ in its prediction computation.

The experimental validation of CAP reveals opportunities for further investigation. CAP has been validated for NUMA-based servers, built on AMD Operton processors and Intel Xeon processors with Nehalem architecture; it requires validation on other architectures, like NVIDA GPU processors and IBM Cell BE processors. Further studies on the power and thermal envelope of multichip server systems, which involve network traffic and off-chip synchronization traffic, is required to understand their contributions to the system thermal envelope.

\section{CONCLUSION}

A fast and accurate model for energy consumption and thermal envelope in a server is critical to understanding and solving the power management challenges unique in dense servers. In this article, we have introduced a comprehensive model of energy consumption by servers as a continuous system of differential equations. The model measures energy input to the system as a function of the work done for completing tasks being gauged and the residual thermal energy given off by the system as a result. Traffic on the system bus, misses in the L2 cache, CPU temperatures, and 
ambient temperatures are combined together to create a model, which can be employed to manage the processor thermal envelope.

The model serves as a predictive tool by approximating observed performance metrics in a discrete time series for estimating future metrics, and thus corresponding energy consumption amounts. It was found through experimental validation that commonly used techniques of regressive time series forecasting, while attractive because of their simplicity, inadequately capture the nonlinear and chaotic dynamics of metric readings for typical server systems. Therefore, a chaotic time series approximation for runtime power consumption is adopted to arrive at Chaotic Attractor Prediction (CAP), which exhibits polynomial time complexity. Our proposed model is the first step towards building solutions for power and thermal management in data centers usually housing many servers.

\section{APPENDIX}

This appendix describes three regression-based prediction models: a linear AR(1) model [Box et al. 1994], a MARS model [Friedman 1991], and an EWMA model. Each model is an approximations to the dynamic system in Equation (4), following regressive combinations of five energy contributors to a server, as given in Equation (7) [Lewis et al. 2008].

The same datasets used to generate the chaotic model were used to create the AR(1) model. Two methods were considered for consolidation: arithmetic mean (average) and geometric mean. Trial models were constructed using each method and a statistical analysis of variance was performed to determine which model generated the best fit to the collected data with a time interval $t=5$ seconds. Note that the statistical coefficients need to be computed only once using some benchmarks, for a given server architecture. The coefficients obtained can then be provided through either the system firmware or the operating system kernel for use in the server executing any application.

Under linear auto-regression, energy consumed by the processor for the AMD server, $E_{\text {proc }}^{A M D}$ as defined by Equation (5), is a linear combination of $M P_{\text {proc }}^{A M D}$ measures (stated in Section 4.1) as [Lewis et al. 2008]:

$$
E_{\text {proc }}^{A M D} \approx 0.49 * T_{C_{0}}+0.50 * T_{C_{1}}+0.01 * H T_{1} .
$$

For the Intel server, its energy consumption by the processor, $E_{\text {proc }}^{\text {Intel }}$, is a function of $M P_{\text {proc }}^{\text {Intel }}$ (detailed in Section 4.1), leading to its estimated energy as follows:

$$
E_{\text {proc }}^{\text {Intel }} \approx 2.29 * T_{C_{0}}+0.03 * T_{C_{1}}+0.52 * Q P L_{C} .
$$

In a similar fashion, energy consumed by the memory subsystem in the AMD server, $E_{m e m}^{A M D}$, is a function of $M P_{\text {mem }}^{A M D}$, yielding

$$
E_{m e m}^{A M D} \approx 0.01 * H T_{2}+0.003 * C M_{0}+0.003 * C M_{1}+0.014 * C M_{2}+0.01 * C M_{3} .
$$

Energy consumption for the memory subsystem in the Intel server, $E_{\text {mem }}^{\text {Intel }}$, is a function of $M P_{\text {mem }}^{\text {Intel }}$, giving rise to

$$
E_{\text {mem }}^{\text {Intel }} \approx 0.52 * Q P L_{I O}+0.35 * C M_{0}+0.31 * C M_{1} .
$$

Energy consumed as a result of disk activities in the AMD server (or the Intel server) is a function of $M P_{h d d}^{A M D}$ (or $M P_{h d d}^{I n t e l}$ ), arriving at

$$
E_{h d d}^{A M D} \approx 0.014 * D_{r}+0.007 * D_{w}
$$

and

$$
E_{\text {hdd }}^{\text {Intel }} \approx 0.01 * D_{r}+0.01 * D_{w}
$$


Energy consumed by the board in the AMD server is a function of $M P_{b o a r d}^{A M D}$, whose components are added in a linearly weighted fashion to derive $E_{\text {board }}^{A M D}$ (or $E_{\text {board }}^{\text {Intel }}$ ), as follows:

$$
E_{\text {board }}^{A M D} \approx 0.101+0.81 * T_{A_{0}}+0.62 * T_{A_{1}}
$$

and

$$
E_{\text {board }}^{\text {Intel }} \approx 2.53+0.03 * T_{A_{0}}+0.01 * T_{A_{1}}+0.01 * T_{A_{2}} .
$$

Finally, energy consumed by electromechanical elements in the AMD server, $E_{e m}^{A M D}$, is a linear function of $M P_{e m}^{A M D}$, leading to

$$
E_{e m}^{A M D} \approx 0.001 * F_{C}+0.001 * F_{M} .
$$

Similarly, energy consumption attributed to electromechanical elements in the Intel server, $E_{\text {em }}^{\text {Intel }}$, equals

$$
\begin{aligned}
E_{\text {em }}^{\text {Intel } \approx 4.85 * F_{C}}+ & 6.61 * F_{M 2 a}+3.92 * F_{M 2 b}+0.28 * F_{M 3 a}+0.52 * F_{M 3 b} \\
& +0.01 * F_{M 4 a}+0.01 * F_{M 4 b}+0.78 * F_{M 5 a}+0.61 * F_{M 5 b} .
\end{aligned}
$$

Total energy consumption for the AMD server (or the Intel server) under AR(1) equals the summation of above five consumption contributors [Lewis et al. 2008].

The models for AMD and Intel severs reveal the issues of adopting linear regression to obtain individual component contribution. Consider the Intel processor as an example. The coefficients for temperature sensors are significantly larger than those for the workload-related $\mathrm{PeCs}$, with those coefficients apparently overbalancing the remaining model components. This fact is quite nonintuitive, as one would like to derive certain physical interpretation on each constant to understand the behavior of its associated model component. In addition, other processor models have negative coefficients for similar model components, making linear regression deemed unsuitable for such modeling [Bertran et al. 2010; McCullough et al. 2011].

The MARS predictor used in our evaluation was created using the consolidated data set employed to establish the CAP and the AR(1) predictors. It was generated by means of the ARESlab toolkit [Jekabsons 2010], and the resulting set of splines served as a predictive tool, as described in Section 5. Note that ARESlab is a MATLAB toolkit for building piecewise-linear and piecewise-cubic MARS regression models. The toolkit was adopted to build a piece-wise cubic model using the same consolidated training set employed for creating the AR and the CAP models. The toolkit output is a structure that defines the basis functions and associated coefficients of Equation (1) given in Section 2 to approximate system dynamics.

The EWMA predictor used in our evaluation was also created using the consolidated dataset employed to established CAP, AR(1), and MARS predictors. The predictor was generated using an Exponentially Weighted Moving Average using the recurrence relation of

$$
\begin{aligned}
& S_{1}=Y_{1} \\
& \left.S_{t}=\alpha Y_{t-1}+(1-\alpha)\right) S_{t-1}, t>1,
\end{aligned}
$$

where $Y_{t}$ is an observation of the power consumed at time $t, S_{t}$ is the value of the weighted average at time $t$, and $\alpha$ is a coefficient representing the weighting decrease degree, for $0 \leq \alpha \leq 1$.

\section{REFERENCES}

AMD. 2006. BIOS and Kernel Developer's Guide for AMD Athlon 64 and AMD Opteron Processors 26094 Rev 3.30 Ed. AMD. 
AMD. 2007. AMD Opteron Processor Data Sheet 3.23 Ed. AMD.

AMD. 2008. Software Optimization Guide for AMD Family 10h Processors 3.0608 Ed. AMD.

Bellosa, F., Weissel, A., Waitz, M., and Kellner, S. 2003. Event-driven energy accounting for dynamic thermal management. In Proceedings of the Workshop on Compilers and Operating Systems for Low Power.

Bertran, R., Gonzalez, M., Martorell, X., Navarro, N., and Ayguade, E. 2010. Decomposable and responsive power models for multicore processors using performance counters. In Proceedings of the 24th ACM International Conference on Supercomputing (ICS '10). ACM, New York, NY, 147-158.

Bhattacharjee, A. And Martonosi, M. 2009. Thread criticality predictors for dynamic performance, power, and resource management in chip multiprocessors. In Proceedings of the 36th International Symposium on Computer Architecture. 290-301.

Bienia, C. 2011. Benchmarking modern multiprocessors. Ph.D. thesis, Princeton University.

Bircher, W. AND John, L. 2007. Complete system power estimation: A trickle-down approach based on performance events. In Proceedings of the IEEE International Symposium on Performance Analysis of Systems and Software. 158-168.

BIRCHER, W. AND JoHn, L. 2012. Complete system power estimation using processor performance events. IEEE Trans Comput. 61, 4, 563-577.

Bircher, W., LAW, J., VAlluRI, W., AND John, L. 2004. Effective use of performance monitoring counters for run-time prediction of power. Tech. Rep. TR-041104-01, University of Texas at Austin.

Bowman, A. And Azzalini, A. 1997. Applied Smoothing Techniques for Data Analysis: The Kernel Approach with S-Plus Illustrations. Oxford University Press.

Box, G., Jenkins, G., And Reinsel, G. 1994. Time Series Analysis, Forecasting and Control. Prentice Hall.

Brochard, L., Panda, R., and Vemuganti, S. 2010. Optimizing performance and energy of HPC applications on POWER7. Comput. Sci. Resear. Dev. 25, 135-140.

Cisco Systems. 2010. Using SPEC CPU2006 benchmark results to compare the compute performance of servers. white paper.

Contreras, G. AND Martonosi, M. 2005. Power prediction for Intel XScale R processors using performance monitoring unit events. In Proceedings of the 2005 International Symposium on Low Power Electronics and Design. ACM, New York, NY, 221-226.

Coskun, A. K., Rosing, T. S., And Gross, K. C. 2008. Proactive temperature balancing for low cost thermal management in MPSoCs. In Proceedings of the IEEE/ACM International Conference on Computer-Aided Design. 250-257.

Davis, J., Rivoire, S., Goldszmidt, M., And Ardestani, E. 2011. Accounting for variability in large-scale cluster power models. In Proceedings of the 2nd Exascle Evaluation and Research Techniques Workshop.

Economou, D., Rivoire, S., Kozyrakis, C., and Ranganathan, P. 2006. Full-system power analysis and modeling for server environments. In Proceedings of the Workshop on Modeling Benchmarking and Simulation.

Electronic Educational Devices, Inc. 2006. WattsUp Power Meter.

Fan, J. And GiJbels, I. 1996. Local Polynomial Modeling and Its Applications. Chapman \& Hall, London, UK.

FAN, J. AND YaO, Q. 2005. Nonlinear Time Series. Springer.

Fan, X., Weber, W.-D., AND Barroso, L. A. 2007. Power provisioning for a warehouse-sized computer. In Proceedings of the 34th International Symposium on Computer Architecture. 13-23.

Friedman, J. 1991. Multivariate adaptive regression splines. Ann. Statist. 19, 1-142.

Gurumurthi, S., Sivasubramaniam, A., And Natarajan, V. 2005. Disk drive roadmap from the thermal perspective: A case for dynamic thermal management. In Proceedings of the 32nd International Symposium on Computer Architecture. 38-49.

Hamill, D., Deane, J., And Aston, P. 1997. Some applications of chaos in power converters. In IEEE Colloquium on Update on New Power Electronic Techniques (Digest No: 1997/091).

Heath, T., Centeno, A. P., George, P., Ramos, L., Jaluria, Y., and Bianchini, R. 2006. Mercury and Freon: Temperature emulation and management for server systems. In Proceedings of the 12th International Conference on Architectural Support for Programming Languages and Operating Systems. ACM, New York, NY, 106-116.

Heath, T., Diniz, B., CARrera, E. V., JR., W. M., AND BiAnchini, R. 2005. Energy conservation in heterogeneous server clusters. In Proceedings of the 10th ACM SIGPLAN Symposium on Principles and Practice of Parallel Programming. 186-195.

Henning, J. L. 2006. SPEC CPU2006 benchmark descriptions. Comput. Archit. News 34, 4.

Hsu, C.-H. AND Poole, S. 2011. Power signature analysis of the SPECpower ssj2008 benchmark. In Proceedings of the IEEE International Symposium on Performance Analysis of Systems and Software. 227-236. 
Hypertransport Technology Consortium. 2007. HyperTransport I/O Link Specification. Specification 3.00c, HyperTransport Technology Consortium.

INTEL. 2009. Intel R64 and IA-32 Architectures Optimization Reference Manual. Intel Corporation.

Isci, C. AND Martonosi, M. 11-15 Feb. 2006. Phase characterization for power: Evaluating control-flowbased and event-counter-based techniques.In Proceedings of the 12th International Symposium on HighPerformance Computer Architecture. 121-132.

Isci, C. AND Martonosi, M. 2003a. Identifying program power phase behavior using power vectors. In Proceedings of the IEEE International Workshop on Workload Characterization. 108-118.

Isci, C. AND Martonosi, M. 2003b. Runtime power monitoring in high-end processors: Methodology and empirical data. In Proceedings of the 36th IEEE/ACM International Symposium on Microarchitecture. 93-104.

Iтон, K. 1995. A Method for Predicting Chaotic Time-seriesWith Outliers. Electronics and Communications in Japan, Part 3: Fundamental Electronic Science 78, 5, 1529-1536.

JeKabsons, G. 2010. ARESLab: Adaptive regression splines toolbox for Matlab. http://www.cs.rtu. lv/jekabsons/regression.html.

Kadayif, I., Chinoda, T., Kandemir, M., Vijaykirsnan, N., Irwin, M., And Sivasubramaniam, A. 2001. vEC: Virtual energy counters. In Proceedings of the ACM SIGPLAN-SIGSOFT Workshop on Program Analysis for Software Tools and Engineering. ACM, 28-31.

Kansal, A., Zhao, F., LiU, J., Kothari, N., AND Bhattacharya, A. A. 2010. Virtual machine power metering and provisioning. In Proceedings of the 1st ACM Symposium on Cloud Computing (SoCC '10). ACM, New York, NY, 39-50.

Kumar, A., Shang, L., Peh, L.-S., And Jha, N. 2008. System-level dynamic thermal management for highperformance microprocessors. IEEE Trans. Comput.-Aid. Des. Integr. Circ. Syst. 27, 1, 96-108.

LEE, K.-J. AND SKADRon, K. 2005. Using performance counters for runtime temperature sensing in highperformance processors. In Proceedings of the 19th IEEE International Symposium Parallel and Distributed Processing.

Lewis, A., GHosh, S., AND Tzeng, N.-F. 2008. Run-time energy consumption estimation based on workload in server systems. In Proceedings of the Workshop on Power Aware Computing and Systems (Hotpower'08).

Lewis, A., Simon, J., AND Tzeng, N.-F. 2010. Chaotic attractor prediction for server run-time energy consumption. In Proceedings of the Workshop on Power Aware Computing and Systems (Hotpower'10).

LiU, S., Memik, S. O., Zhang, Y., AND Memik, G. 2008. A power and temperature aware DRAM architecture. In Proceedings of the 45th Annual Design Automation Conference (DAC'08). ACM, New York, NY, 878-883.

Liu, S., Memiк, S. O., Zhang, Y., And Memiк, G. 2008. A power and temperature aware DRAM architecture. In Proceedings of the 45th Annual Design Automation Conference (DAC'08). ACM, New York, NY, 878-883.

Liv, Z. 2010. Chaotic time series analysis. Math. Problems Engin.

London, K., Moore, S., Mucci, P., Seymour, K., and Luczak, R. 2001. The PAPI cross-platform interface to hardware performance counters. In Department of Defense Users' Group Conference Proceedings.

McCullough, J. C., Agarwal, Y., Chandrashekar, J., Kuppuswamy, S., Snoeren, A. C., and Gupta, R. K. 2011. Evaluating the effectiveness of model-based power characterization. In Proceedings of the USENIX Annual Technical Conference.

Mesa-Martinez, F., Nayfach-Battilana, J., and Renau, J. 2007. Power model validation through thermal measurements. In Proceedings of the 34th International Conference on Computer Architecture. 302-311.

Mesa-Martinez, F. J., Ardestani, E. K., and Renau, J. 2010. Characterizing processor thermal behavior. SIGARCH Comput. Archit. News 38, 193-204.

Micron, Inc. 2007. Calculating Memory System Power for DDR3. Tech. Note TN41 01DDR3 Rev.B, Micron, Inc.

NIST. 2010. NIST/SEMATECH e-Handbook of Statistical Methods.

Phansalkar, A., Joshi, A., AND John, L. K. 2007. Analysis of redundancy and application balance in the SPEC CPU2006 benchmark suite. SIGARCH Comput. Archit. News 35, 412-423.

Powell, M., Biswas, A., Emer, J., Mukherjee, S., Sheikh, B., and Yardi, S. 2009. CAMP: A technique to estimate per-structure power at run-time using a few simple parameters. In Proceedings of the IEEE 15th International Symposium on High Performance Computer Architecture. 289-300.

Reich, J., Goraczko, M., Kansal, A., And PadHye, J. 2010. Sleepless in Seattle no longer. In Proceedings of the USENIX Annual Technical Conference.

RIVOIRE, S. 2008. Models and metrics for energy-efficient computer systems. Ph.D. thesis, Stanford University. Rivoire, S., Ranganathan, P., and Kozyrakis, C. 2008. A comparison of high level full-system power models. In Proceedings of the USENIX Workshop on Power Aware Computing and Systems. 
Server System Infrastructure Consortium. 2004. EPS12v Power Supply Design Guide, V2.92. Spec. 2.92, Server System Infrastructure Consortium.

Singh, K., Bhadauria, M., And McKee, S. A. 2009. Real time power estimation and thread scheduling via performance counters. SIGARCH Comput. Archit. News 37, 2, 46-55.

Skadron, K., Stan, M. R., Sankaranarayanan, K., Huang, W., Velusamy, S., and Tarjan, D. 2004. Temperatureaware Microarchitecture: Modeling and Implementation. ACM Trans. Archit. Code Optim. 1, 1, 94-125.

Sprott, J. 2003. Chaos and Time-Series Analysis. Oxford University Press, New York, NY.

Su, L.-Y. 2010. Prediction of multivariate chaotic time series with local polynomial fitting. Comput. Math. App.s 59, 2, 737-744.

Sun Microsystems. 2008. Solaris System Administration Guide: Advanced Administration.

Ton, M., Fortenbery, B., And Tschudi, W. 2008. DC power for improved data center efficiency. Tech. rep., Lawrence Berkeley National Laboratory.

Tong, H. 1993. Non-Linear Time Series: A Dynamical System Approach. Oxford University Press, New York, NY.

Tse, C. And Di Bernardo, M. 2002. Complex Behavior In Switching Power Converters. Proc. IEEE 90, 5, 768-781.

Tsirogiannis, D., Harizopoulos, S., And Shah, M. A. 2010. Analyzing the energy efficiency of a database server. In Proceedings of the International Conference on Management of Data. (SIGMOD '10). ACM, New York, NY, 231-242.

TuRLACH, B. 1993. Bandwidth selection in kernel density estimation: A review. CORE and Institut de Statistique. 23-493.

Varsamopoulos, G., AbBasi, Z., And Gupta, S. 2010. Trends and effects of energy proportionality on server provisioning in data centers. In Proceedings of the International Conference on High Performance Computing. 1-11.

Ware, M., Rajamani, K., Floyd, M., Brock, B., Rubio, J., Rawson, F., And Carter, J. 2010. Architecting for power management: The IBM POWER7 approach. In Proceedings of the IEEE 16th International Symposium on High Performance Computer Architecture. 1-11.

Received October 2010; revised August 2011, December 2011; accepted May 2012 